\title{
THE OSMOPROTECTIVE EFFECT OF SOME ORGANIC SOLUTES ON STREPTOMYCES SP. MADO2 AND NOCARDIOPSIS SP. MADO3 GROWTH
}

\author{
Hanane Ameur ${ }^{1 *}$, Mostefa Ghoul ${ }^{1}$, Joseph Selvin ${ }^{2}$ \\ ${ }^{1}$ Laboratoire d'écologie microbienne, Département de Biologie, Faculté des sciences, Université Ferhat Abbas, Sétif 19000, \\ Algeria; ${ }^{2}$ Marine Bioprospecting Laboratory, Department of Microbiology, Bharathidasan University, Tiruchirappalli 620024 , \\ India.
}

Submitted: March 12, 2010; Returned to authors for corrections: May 19, 2010; Approved: November 04, 2010.

\begin{abstract}
The response of two marine actinomycetes such as Streptomyces sp. MADO2 and Nocardiopsis sp. MADO3 to osmotic stress in minimal medium M63 and in glycerol-asparagine medium (ISP5) was studied. The two strains were moderately halophilic and the behavior of the strain Streptomyces sp. MADO2 and Nocardiopsis sp. MADO3 towards the salt stress was varied depends on the media composition and the salinity concentration. The strain Streptomyces sp. was more sensitive to salt stress than Nocardiopsis sp. The growth of both Streptomyces sp. and Nocardiopsis sp. were inhibited at $1 \mathrm{M} \mathrm{NaCl}$ irrespective of the medium used. The Nocardiopsis sp. acquired osmoadaptation on ISP5 medium whereas the Streptomyces sp. showed poor growth on M63 medium. Glycine betaine (GB), proline and trehalose played a critical role in osmotic adaptation at high osmolarity whereas at low osmolarity they showed an inhibitory effect on the bacterial growth. The present findings confirmed that GB was the powerful osmoprotectant for Streptomyces sp. and Nocardiopsis sp. grown at $1 \mathrm{M} \mathrm{NaCl}$ both in M63 and ISP5 media.
\end{abstract}

Key words: Streptomyces sp., Nocardiopsis sp., osmoprotective effect, M63 medium, ISP5 medium.

\section{INTRODUCTION}

Actinomycetes are potential producers having immense application in industrial production processes. Their capacity to degrade complex organic compounds and to produce antibiotics has been demonstrated (1). Halophilic or salt tolerant actinomycetes are being developed as model organisms to disclose the mechanism and microbial physiology under extreme environments (3). Microorganisms found in extreme environments have attracted a great attention due to their production of various natural compounds and their specialized mechanisms for adaptation to extreme environments (28). However, relatively little information was available on the osmoregulatory strategy of actinomycetes, even though needs a special attention because of their transitional nature between the simple eubacteria and the fungi $(13,19)$.

Most microorganisms subjected to water stress accumulate

\footnotetext{
*Corresponding Author. Mailing address: Laboratoire d'écologie microbienne, Département de Biologie, Faculté des sciences, Université Ferhat Abbas, Sétif 19000, Algeria..; E-mail: ameur_han@yahoo.fr
} 
organic solutes to control their internal water activity, maintain the appropriate cell volume and turgor pressure, and protect intracellular macromolecules (8). Prokaryotes have developed two strategies to cope with increasing salinities. One is to accumulate and adjust the internal concentration of inorganic ions such as $\mathrm{K}^{+}$and $\mathrm{Cl}^{-}$to values that counteract the external osmolarity (25). A different strategy is the accumulation of osmolytes or compatible solutes including sugars (trehalose), free amino acids (e.g., glutamate and proline), and quaternary ammonium compounds (e.g., glycine betaine, proline betaine, butyrobetaine, and carnitine) that bacteria accumulate from de novo synthesis or from externally provided osmoprotectants such as choline, the precursor of glycine betaine $(19,6,16$, 18).

The present study aims to analyze the effect of increasing osmolarity of $\mathrm{NaCl}$ (0-1 M) on the growth of Streptomyces sp. and Nocardiopsis sp. isolated from the marine sponge Fasciospongia cavernosa in minimal medium M63 and in glycerol-asparagine medium (ISP5 medium). It was a maiden initiative to use M63 medium to analyze the effect of increased salinity and osmoprotectants on the growth of actinomycetes strains. This minimal medium is generally used for the study of halotolerance of enteric bacteria. The osmoprotective effect of glycine betaine (GB), proline and trehalose was also studied.

\section{MATERIALS AND METHODS}

\section{Bacterial strains and antimicrobial screening}

The marine actinomycetes such as Streptomyces sp. MADO2 and Nocardiopsis sp. MADO3 were obtained from marine bioprospecting laboratory, Bharathidasan University, Tiruchirappalli (India). Primary screening of antimicrobial activities was performed on Actinomycetes agar (Himedia) supplemented with $2 \% \mathrm{NaCl}$. The screening of the isolates was examined against $C$. albicans PC1, E. coli PC2, P. mirabilis PC3, S. haemolytic. PC4, P. aeruginosa PC5, M. luteus PC6, S. epidermidis PC7, E. faecalis PC8, K. pneumoniae PC9, B. subtilis $\mathrm{PC} 10$ and $S$. aureus PC11.

\section{Medium and cultivating conditions}

YM medium (19), containing $10 \mathrm{~g}$ of malt extract, $4 \mathrm{~g}$ of yeast extract, $0.01 \mathrm{~g}$ of $\mathrm{CaCl}_{2}$ and $1 \mathrm{ml}$ of trace salts solution (per $100 \mathrm{ml}$ of water: $100 \mathrm{mg}$ of $\mathrm{FeSO}_{4} \cdot 7 \mathrm{H}_{2} \mathrm{O}, 100 \mathrm{mg}$ of $\mathrm{MnCl}_{2} \cdot 4 \mathrm{H}_{2} \mathrm{O}, 100 \mathrm{mg}$ of $\mathrm{ZnSO}_{4} \cdot \mathrm{H}_{2} \mathrm{O}$ and $100 \mathrm{mg}$ of $\mathrm{CaCl}_{2}$ ) was used for the preparation of the inoculum of the strains MADO2 and MADO3. Cells were grown aerobically for $72 \mathrm{~h}$ at $26^{\circ} \mathrm{C}$ on a rotary shaking incubator at $250 \mathrm{rpm}$. After centrifugation and washing with $0.9 \% \mathrm{NaCl}$ solution, $1 \mathrm{ml}$ of cell suspension served as the inoculum for $50 \mathrm{ml}$ of culture in M63 and in ISP5 medium respectively. The inoculum contained $4.2 \times 10^{7} \mathrm{cfu} / \mathrm{ml}$.

\section{Effect of increased salinity on the bacterial growth}

For $\mathrm{NaCl}$ endurance experiments, M63 medium $[(\mathrm{g} / \mathrm{l})$ $\mathrm{KH}_{2} \mathrm{PO}_{4} 13.6 \mathrm{~g}, \mathrm{KOH} 4.2 \mathrm{~g},\left(\mathrm{NH}_{4}\right)_{2} \mathrm{SO}_{4} 1.982 \mathrm{~g}, \mathrm{MgSO}_{4} .7 \mathrm{H}_{2} \mathrm{O}$ $0.246 \mathrm{~g}, \mathrm{FeSO}_{4} .7 \mathrm{H}_{2} \mathrm{O} 0.0005 \mathrm{~g}$ and glucose $\left.1.8 \mathrm{~g}(4)\right]$ and ISP5 [(g/l) asparagine $1 \mathrm{~g}$, glycerol $10 \mathrm{~g}, \mathrm{~K}_{2} \mathrm{HPO}_{4} 1 \mathrm{~g}$ and $1 \mathrm{ml}$ of trace salts solution (26)] were used as the basic media. The $\mathrm{NaCl}$ concentrations include: $0 \mathrm{M}, 0.1 \mathrm{M}, 0.5 \mathrm{M}, 0.8 \mathrm{M}$ and 1 $\mathrm{M}$ were enriched in both media.

\section{Osmoprotective effect of GB, proline and trehalose on the bacterial growth}

To study the osmoprotective effect of osmoprotectants described above, the media supplemented with GB (1 $\mathrm{mM})$, proline $(1 \mathrm{mM})$ and trehalose $(1 \mathrm{mM})$ were used. MADO2 and MADO3 strains were cultivated in M63 and ISP5 media aerobically at $26^{\circ} \mathrm{C}$ on a rotary shaking incubator at $100 \mathrm{rpm}$. Bacterial growth was monitored spectroscopically at $540 \mathrm{~nm}$. The number of cells corresponding to the absorbance was counted using a Petroff-Hausser chamber

\section{RESULTS}

\section{Antimicrobial activity}

The two isolates were screened for their antimicrobial activities against the indicator strains as mentioned above. Streptomyces sp. showed a significant antimicrobial activity 
against C. albicans PC1 $(23 \mathrm{~mm})$, E. coli $\mathrm{PC} 2(16 \mathrm{~mm})$ P. $\quad$ showed activity against C. albicans PC1 (14 mm), E. coli PC2 mirabilis PC3 (26 mm), M. luteus PC6 (27 mm) and S. aureus $\quad(00 \mathrm{~mm})$, P. mirabilis PC3 (12 mm), M. luteus PC6 (23 mm) PC11 (26 mm). It was more active than Nocardiopsis sp. and S. aureus PC11 (19 mm) (Table 1).

Table 1. Antimicrobial activity of Streptomyces sp. MADO2 and Nocardiopsis sp. MADO3 on some clinical pathogens

\begin{tabular}{|c|c|c|c|}
\hline \multirow{2}{*}{\multicolumn{2}{|c|}{ Clinical pathogens }} & \multicolumn{2}{|c|}{ Inhibition zone (mm) } \\
\hline & & \multirow{2}{*}{$\frac{\text { Streptomyces sp. MADO2 }}{23}$} & \multirow{2}{*}{$\frac{\text { Nocardiopsis sp.MADO3 }}{14}$} \\
\hline C. albicans & PC1 & & \\
\hline E. coli & PC2 & 16 & 00 \\
\hline P. mirabilis & PC3 & 26 & 12 \\
\hline H. streptococcus & PC4 & 12 & 08 \\
\hline P. aeruginosa & PC5 & 13 & 16 \\
\hline M. luteus & PC6 & 27 & 23 \\
\hline S. epidermidis & PC 7 & 22 & 14 \\
\hline E. faecalis & PC 8 & 16 & 13 \\
\hline K. pneumoniae & PC 9 & 08 & 11 \\
\hline B. subtilis & PC 10 & 23 & 22 \\
\hline S. aureus & PC 11 & 26 & 19 \\
\hline
\end{tabular}

\section{Growth characteristics of strains MADO2 and MADO3 in}

\section{M63 and ISP5 media}

The growth characteristics of Streptomyces sp. was compared with those of Nocardiopsis sp. using M63 and ISP5 media (Fig.1). The growth of Streptomyces sp. was favored irrespective of the media used. However, Nocardiopsis sp. growth was better in M63 medium than in ISP5 medium.


Figure 1. Growth characteristics of Streptomyces sp. MADO2 (A) and Nocardiopsis sp. MADO3 (B) grown in M63 medium and ISP5 medium. 


\section{Effect of increased osmolarity on the bacterial growth}

To analyze the response of Streptomyces $\mathrm{sp}$. and Nocardiopsis sp. to increased osmolarity, cells were cultivated in M63 and ISP5 media containing 0-1 M NaCl. Exposure to elevated osmotic strength reduced the growth of Streptomyces sp. At $0.5 \mathrm{M} \mathrm{NaCl}$, the growth rate decreased significantly in both M63 and ISP5 media (Fig. 2). The effect of increased salinity on the growth of Nocardiopsis sp. was different to that of Streptomyces sp. The increase in growth occurred beyond $0.5 \mathrm{M} \mathrm{NaCl}$, a drastic decline of growth was observed (Fig. 3).
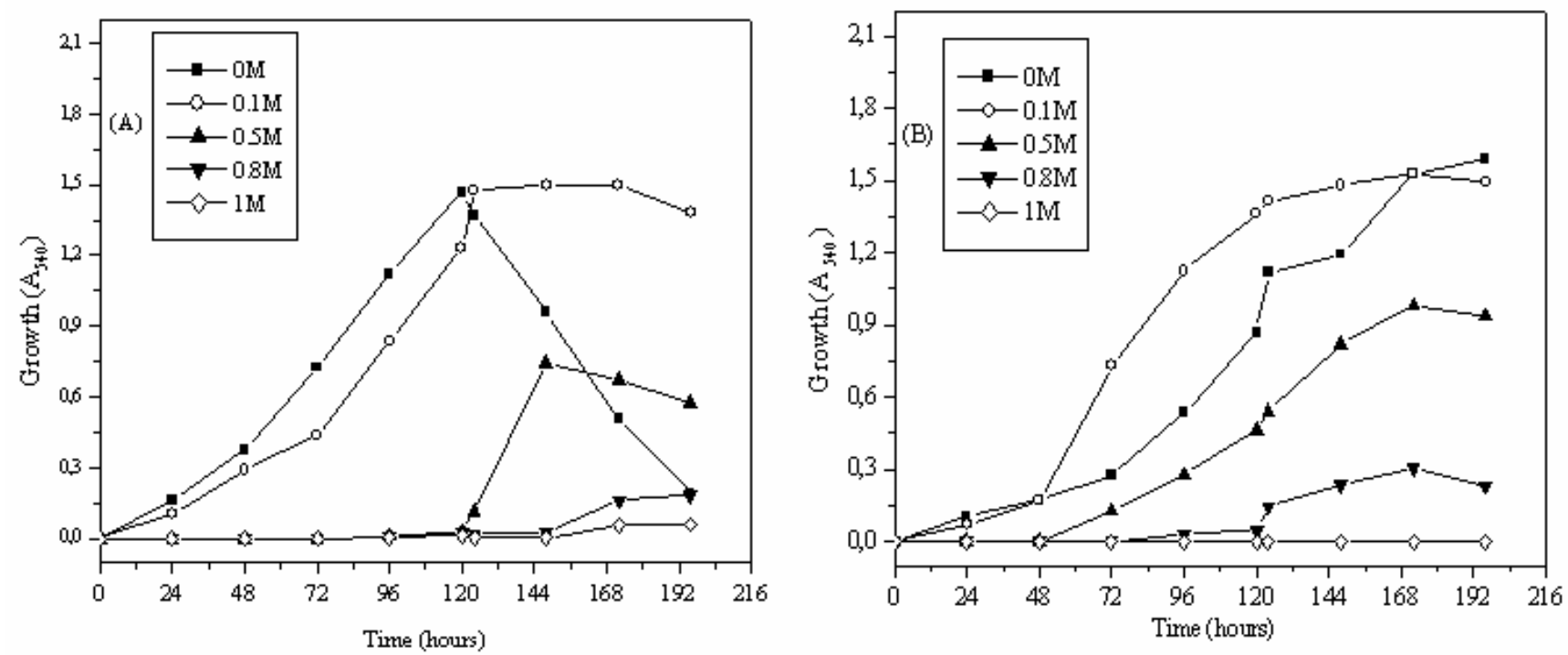

Figure 2. Growth of Streptomyces sp. (MADO2) of increased salinity in: M63 medium (A) and in ISP5 medium (B).
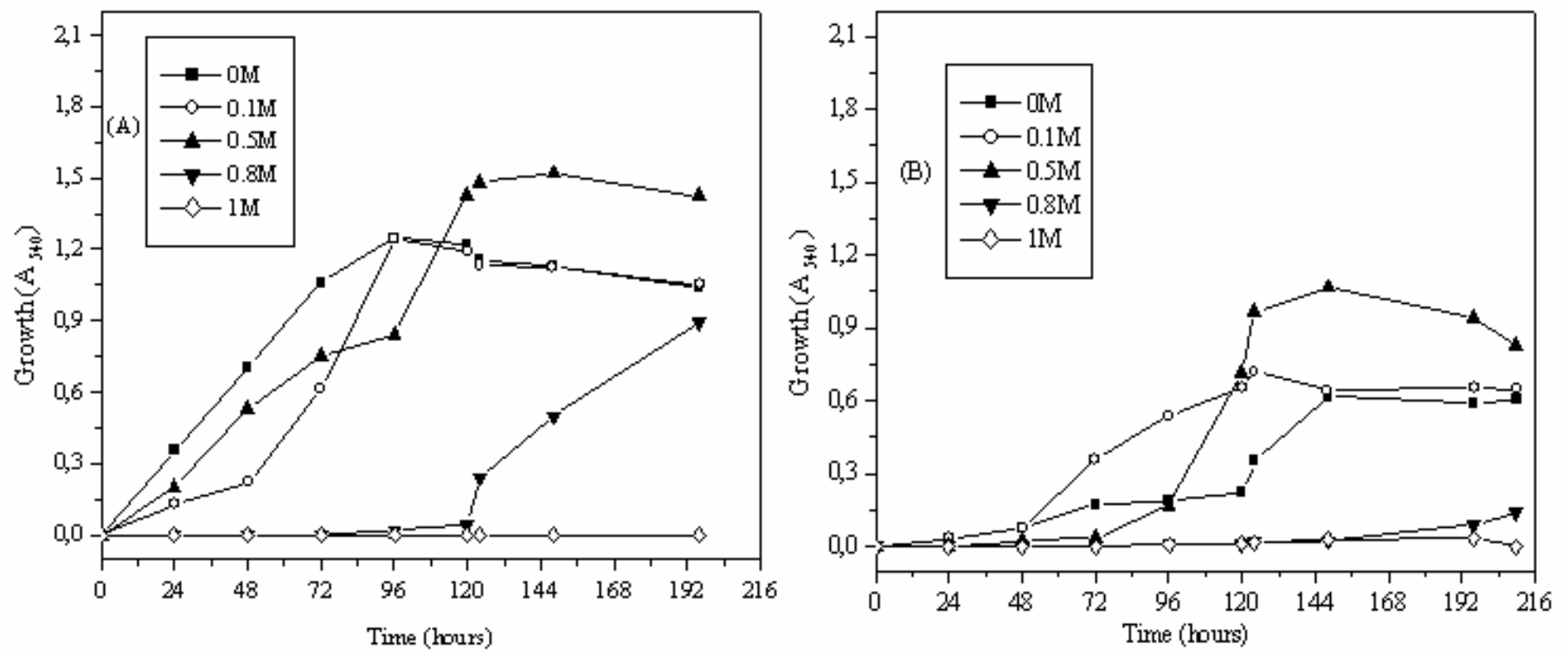

Figure 3. Growth of Nocardiopsis sp. (MADO3) of increased salinity in: M63 medium (A) and in ISP5 medium (B). 
Effect of GB, proline and trehalose on the bacterial growth at different concentrations of $\mathrm{NaCl}$

At $0.1 \mathrm{M} \mathrm{NaCl}$, the supplementation of trehalose in M63 medium enhanced the growth of Streptomyces sp. whereas GB and proline showed an inhibitory effect on the growth. The condition was found to be entirely different in the case of
Nocardiopsis sp. where the supplement of GB and proline enhanced the growth.

The ISP5 medium supplemented with osmoprotectants inhibited the growth of Streptomyces sp. whereas the osmoprotectants trehalose and proline stimulated the growth of Nocardiopsis sp. (Fig .4).


Figure 4. Growth of Streptomyces sp. MADO2 in M63 medium (A), in ISP5 medium (B) and Nocardiopsis sp. MADO3 in M63 medium (C) and ISP5 medium (D) in presence of $0.1 \mathrm{M} \mathrm{NaCl}$ and/or GB, proline, trehalose (1 mM).

In presence of $0.5 \mathrm{M} \mathrm{NaCl}$, both $\mathrm{M} 63$ and ISP5 media supplemented with GB, proline and trehalose showed an osmoprotective effect and enhanced the bacterial growth of
Streptomyces sp. Growth of Nocardiopsis sp. was nearly the same in M63 medium with or without GB. Trehalose and proline acted positively on the growth of Nocardiopsis sp. 
However, the growth was reduced in ISP5 medium supplemented with GB and proline. But trehalose stimulated the growth of Nocardiopsis sp. (Fig. 5).

Growth of Streptomyces sp. and Nocardiopsis sp. were greatly increased at $0.8 \mathrm{M} \mathrm{NaCl}$ in $\mathrm{M} 63$ medium supplemented with GB, proline and trehalose. However, it is noteworthy that trehalose was more effective than GB and proline. Nocardiopsis sp. growth was stimulated significantly in ISP5 medium with GB. Trehalose and proline stimulated the growth of Streptomyces sp. Hence, we conclude that trehalose was the potent osomprotectant stimulating the growth of Streptomyces sp. besides GB and proline (Fig. 6).
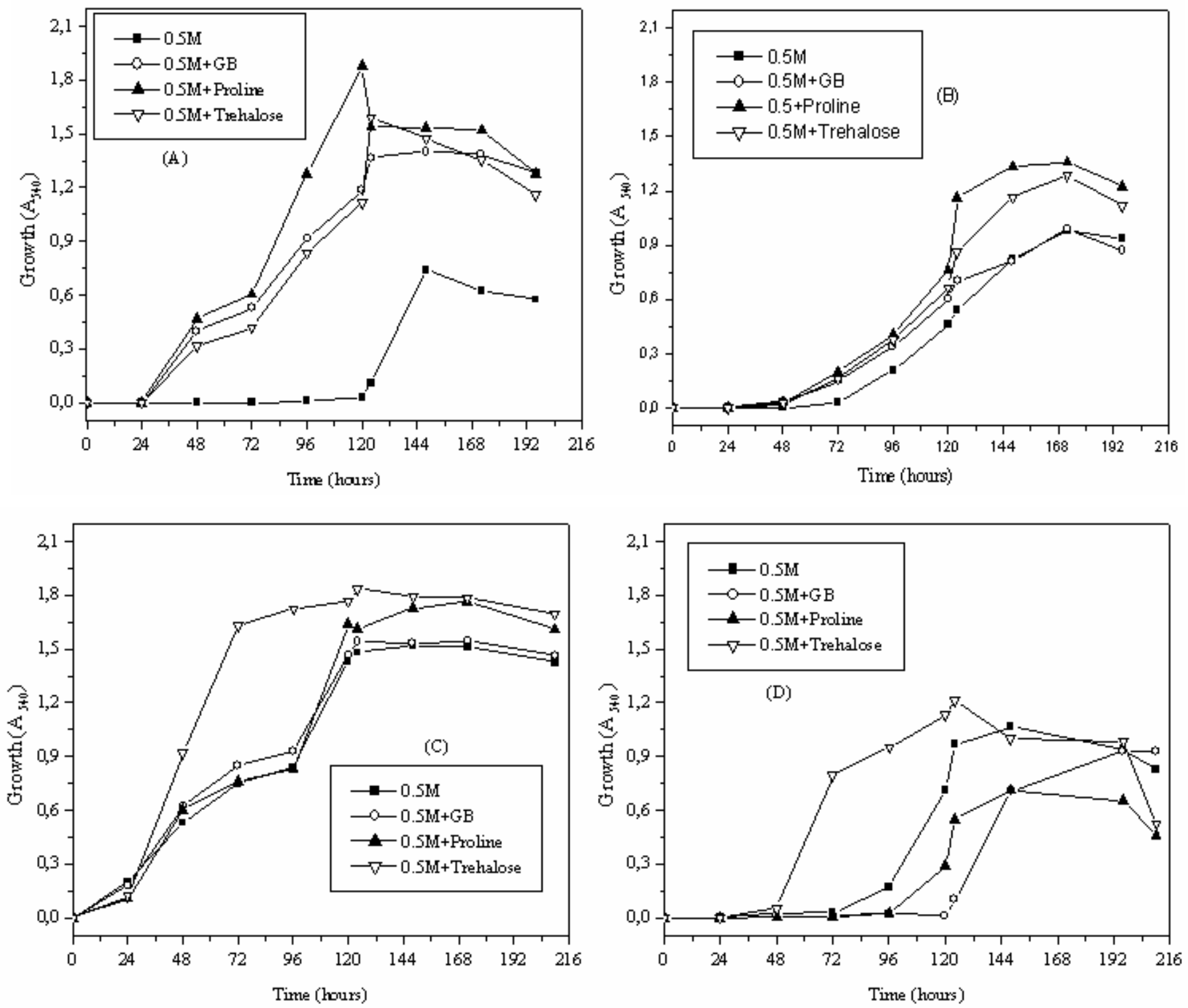

Figure 5. Growth of Streptomyces sp. MADO2 in M63 medium (A), in ISP5 medium (B) and Nocardiopsis sp. MADO3 in M63 medium (C) and ISP5 medium (D) in presence of $0.5 \mathrm{M} \mathrm{NaCl}$ and/or GB, proline, trehalose (1 mM). 

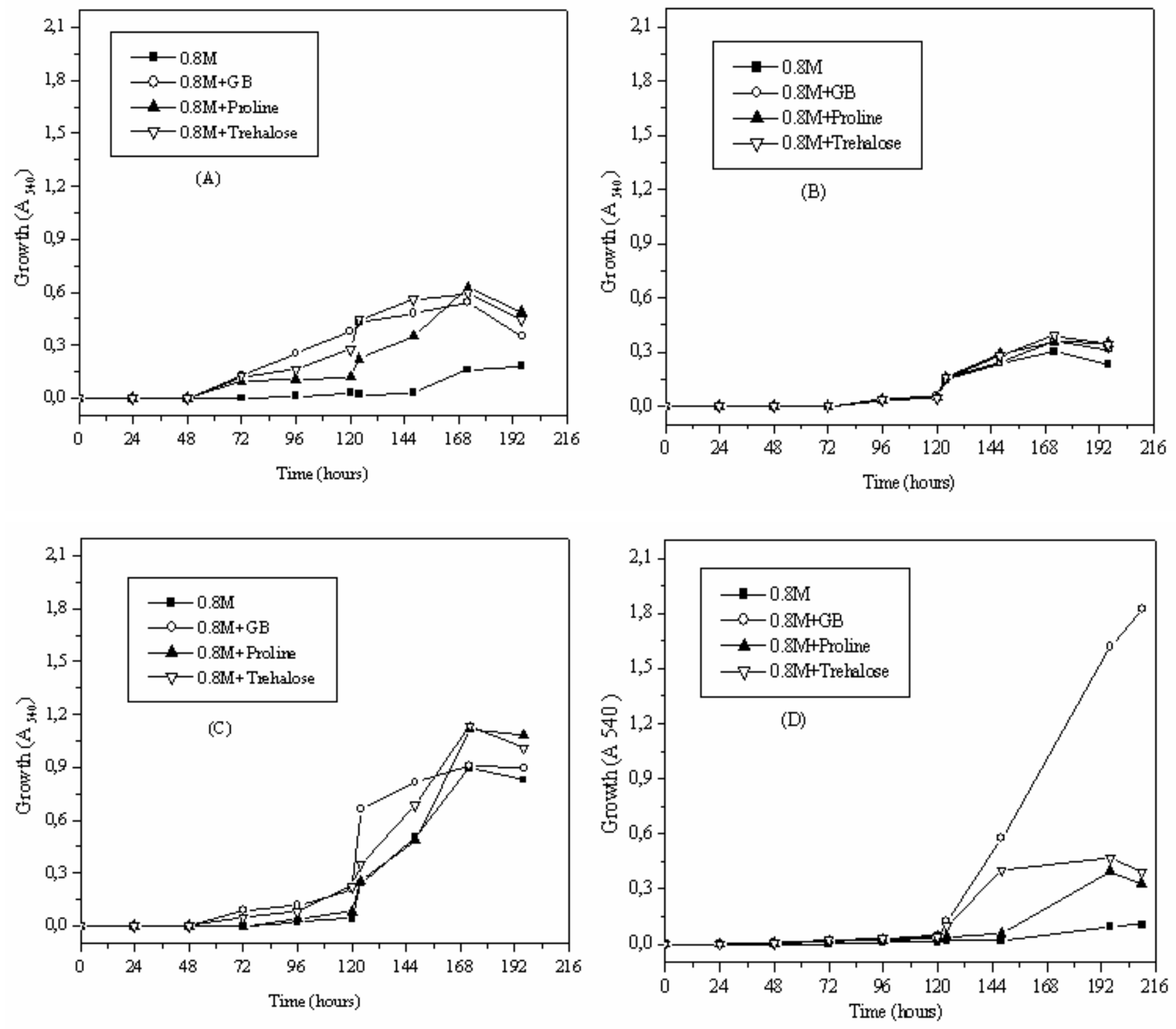

Figure 6. Growth of Streptomyces sp. MADO2 in M63 medium (A), in ISP5 medium (B) and Nocardiopsis sp. MADO3 in M63 medium (C) and ISP5 medium (D) in presence of $0.8 \mathrm{M} \mathrm{NaCl}$ and/or GB, proline, trehalose (1 mM).

In M63 medium supplemented with $1 \mathrm{M} \mathrm{NaCl,} \mathrm{GB}$ was the most efficient osmoprotectant stimulating the growth of Streptomyces sp. and Nocardiopsis sp. in comparison with proline and trehalose. Moreover, little or no growth was observed in Streptomyces sp. and Nocardiopsis sp. respectively without osmoprotectants. In ISP5 medium, GB proved to be a potent osmoprotectant stimulating the growth of Streptomyces sp. However, its osmoprotective effect was negligible for Nocardiopsis sp. (Fig. 7). 

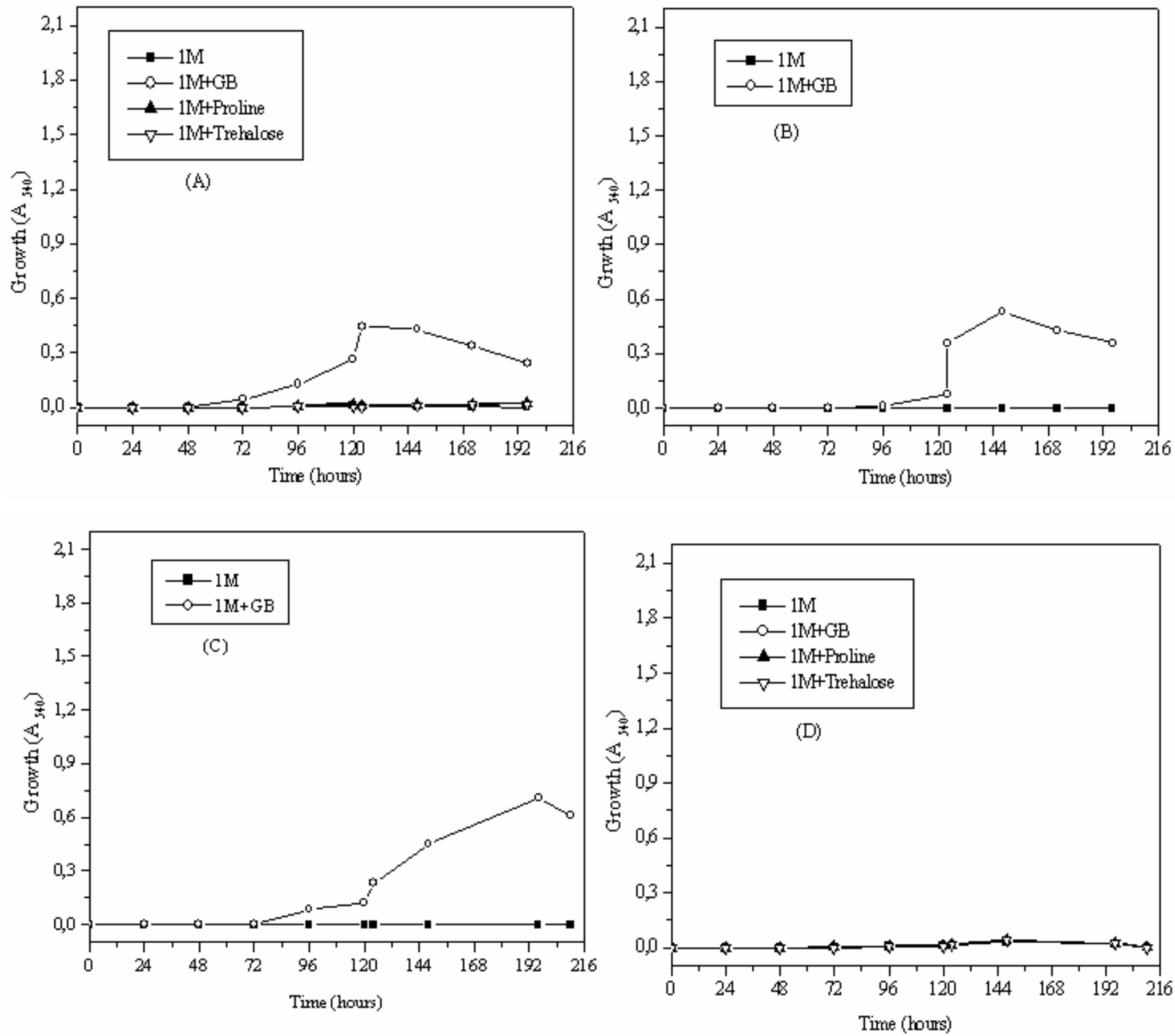

Figure 7. Growth of Streptomyces sp. MADO2 in M63 medium (A), in ISP5 medium (B) and Nocardiopsis sp. MADO3 in M63 medium (C) and ISP5 medium (D) in presence of $1 \mathrm{M} \mathrm{NaCl}$ and/or GB, proline, trehalose (1 $\mathrm{mM})$.

\section{DISCUSSION}

Marine organisms produce enormous antibiotics chemicals. They are emerging as an exciting species for the discovery of new classes of therapeutics and it could provide the drugs needed to sustain us for the next 100 years in our battle against drug resistant infectious diseases (30). Our results showed that the two strains had an antimicrobial activity against the clinical pathogens tested. Nocardiopsis sp. VITSVK5 (FJ973467) isolated from marine sediment samples collected at the Puducherry coast of India showed a significant antibacterial activity against Gram negative bacteria- E. coli 
(20 mm), P. aeruginosa $(18 \mathrm{~mm})$ and $K$. pneumonia $(15 \mathrm{~mm})$ and Gram positive bacteria- E. faecalis $(20 \mathrm{~mm})$, B. cereus $(13$ $\mathrm{mm})$ and $S$. aureus $(6 \mathrm{~mm})$. They also showed an antifungal activity against $A$. fumigatus $(23 \mathrm{~mm})$, A. flavus $(15 \mathrm{~mm})$ and A. niger (12 mm) (29).

It was found that the behavior of the two strains towards salt stress was different depending on the type of the medium used and the degree of salinity. The absence of $\mathrm{NaCl}$ promoted the growth of Streptomyces sp. irrespective of the media used. However, Nocardiopsis sp. growth was better in M63 medium than in ISP5 medium. It was found that Streptomyces sp. utilized both glucose and glycerol as carbon source, while Nocardiopsis sp. utilized glucose as sole carbon source. Growth of Streptomyces clavuligerus strain Mit-1 grown on different media has been studied (11). The growth was very low in ISP5 medium than in the other media like Actinomycete broth (maximal growth) or in Gelatin casaminoacid broth. Moreover, various sugars were investigated in the same study for their effect on the bacterial growth. Glucose was the best carbon source comparative to lactose, maltose, sucrose and xylose. It should be noted that growth of Streptomyces sp. and Nocardiopsis sp. decreased in M63 medium. This is can be explained by the poor composition of this minimal medium which contains only glucose as carbon source. In the presence of increased osmolarity, it was obtained that the two strains are moderately halophilic. Halophilic bacteria and actinomycetes have been grouped according to salinity requirements by microbiologists. The method of classification proposed by Kushner (15) appears to be the most popular one.

Streptomyces sp. was more sensitive to salt stress than Nocardiopsis sp. while Nocardiopsis sp. showed an optimum growth at $0.5 \mathrm{M} \mathrm{NaCl}$. It was observed that Nocardiopsis sp. requires $3 \%$ of $\mathrm{NaCl}$ for its growth while Streptomyces $\mathrm{sp}$. requires $0.58 \%$ of $\mathrm{NaCl}$. At $0.8 \mathrm{M} \mathrm{NaCl}$, Streptomyces sp. growth decreased significantly in M63 medium than in ISP5 medium. It has demonstrated that polyols like glycerol, arabitol and inositol are typical compatible solutes of halophilic/halotolerant fungi, algae, bacteria and plants (7). For Nocardiopsis sp., its growth was considerable in M63 medium than in ISP5 medium. This is can be explained by the fact that Nocardiopsis sp. synthesizes trehalose from glucose, which is an ingredient of M63 medium. We noted that M63 medium contains $10 \mathrm{mM}$ glucose, which allowed the stressed-cells to accumulate trehalose as an osmoprotectant. According to McBride and Ensign (21), spores of Streptomyces griseus grown on DMC medium containing $200 \mathrm{mM}$ glucose contain $21 \%$ of their dry weight as trehalose while spores grown on media containing $20 \mathrm{mM}$ glucose contained $1.2 \%$ of their dry weight as trehalose. The relationship between glucose concentration in the medium and trehalose content of spores was essentially linear between 5 and $100 \mathrm{mM}$ glucose. It has been mentioned that trehalose is widespread disaccharide occurring in eukaryotic and prokaryotic alike. Because they are often observed in slightly halotolerant of marine organisms growing at $3 \% \mathrm{NaCl}(0.5 \mathrm{~mol} / \mathrm{l})$ or just above, a prospective role as osmolytes was assumed (7).

At $1 \mathrm{M} \mathrm{NaCl}$, growth of Streptomyces sp. and Nocardiopsis sp. was totally inhibited in both ISP5 and M63 media. Poor growth of Streptomyces sp. was observed in M63 medium. An osmoadaptation was acquired for Nocardiopsis sp. in ISP5 medium. It seems possible that the two strains accumulated or synthesized osmolytes to regulate response to osmotic stress.

Concerning the osmoprotective effect of GB, proline and trehalose, it was observed that the three components play a critical role in osmotic adaptation at high osmolarity whereas at low osmolarity they may have an inhibitory effect on the bacterial growth. Unfortunately, very little is known about the $\mathrm{NaCl}$ tolerance, the halophilism and the osmoadaptation of actinomycetes. Further research warranted to explore whether actinomycetes respond to osmotic stress in a way similar to halophilic bacteria or not and their compatible solutes are the same nature as observed in eubacteria (31).

Based on the present findings, the GB has an inhibitory 
effect at $0.1 \mathrm{M} \mathrm{NaCl}$ on the growth of Nocardiopsis sp. in ISP5 medium whereas proline and trehalose stimulated its growth. The inhibitory effect of GB, proline and trehalose was also observed in Streptomyces sp. grown in ISP5 medium whereas only GB and proline inhibited the growth of this strain in M63 medium. Nocardiopsis sp. growth was also inhibited in ISP5 medium at 0.5 $\mathrm{M} \mathrm{NaCl}$ by adding GB and proline. However, it should be noted that an osmoprotective effect of GB, proline and trehalose on the growth of Streptomyces sp. both in M63 and ISP5 media at $0.5 \mathrm{M}$ $\mathrm{NaCl}$ was observed. Therefore, it is noteworthy that the inhibitory effect of GB and proline particularly on the bacterial growth depending not only on the salinity range (low osmolarity) rather the effect also duo to the nature of the culture media. The observations concerning the inhibitory effect of GB, proline and trehalose were similar to that of previous reports $(9,12,23)$ which have also demonstrated that GB and other osmoprotectants had an inhibitory effect on growth of Lactococcus lactis.

The osmoprotective effect of GB, proline and trehalose on the two strains has shown only at inhibitory salinities. Proline is the main compatible solute in Streptomyces sp. at $0.5 \mathrm{M}$ $\mathrm{NaCl}$ grown in M63 medium and in ISP5 medium. GB and proline are two major organic osmolytes that accumulate in a variety of plant species (2) and in many microorganisms (5) in response to environmental stresses. In Salmonella oranienburg, exogenous proline could alleviate the growth inhibition imposed by osmotic stress (5). It is also often observed that proline is raised in a salt stress situation as in Streptomyces $\mathrm{sp}$ (7). Furthermore, addition of $12.5 \mathrm{mM}$ proline to the growth medium increased the specific growth yield of Streptomyces griseus (14). At $0.8 \mathrm{M} \mathrm{NaCl}$, trehalose confers an osmoadaptation in Streptomyces sp. in both M63 and ISP5 media. Trehalose stimulates Nocardiopsis sp. growth besides GB and proline. Trehalose is known to stabilize membranes, proteins and whole cell during dehydration and storage. It participates in some way to alleviate osmotic stress and that trehalose is primarily a stress metabolite associated with the onset of unfavorable growth conditions (7). Trehalose is also the major organic osmoprotectant in Rhodobacter sphaeroides f. sp. denitrificans IL 106 (32). Obtained results confirmed that GB was the powerful osmoprotectant in Streptomyces sp. grown at $1 \mathrm{M} \mathrm{NaCl}$ in both M63 and ISP5 media and for Nocardiopsis sp. grown in M63 medium. Glycine betaine has been generally regarded as a superior protective osmolyte for E. coli and has been shown to increase tolerance to sugars, salts and organic acids $(5,22)$. Glycine betaine is of general importance for osmotic adaptation of most eubacteria (10). It is the most efficient osmoprotectant characterized to date (27). The transport and the accumulation of GB are triggered by raising the osmotic pressure of the external environment (17, $24,20)$. The main results proved that the osmoprotective effect of GB, proline and trehalose in Streptomyces sp. and Nocardiopsis sp. was shown only at inhibitory salinities whereas at low osmolarity they had an inhibitory effect on the bacterial growth.

\section{REFERENCES}

1. Al-Zarban, S. S.; Al-Musallam, A. A.; Abbas, I. H.; Fasasi, Y. A. (2002). Noteworthy salt-loving actinomycetes from Kuwait. Kuwait. S. Sci. Eng., 29, 99-109.

2. Ashraf, M.; Foolad, M. R. (2007). Roles of glycine betaine and proline in improving plant abiotic stress resistance. Environmental and Experimental Botany, 59, 206-216.

3. Cai, Y.; Xue, Q.; Chen, Z.; Zhang, R. (2009). Classification and salttolerance of actinomycetes in the Qinghai Lake water and Lakeside saline soil. J. Sust. Dep., 02, 107-110.

4. Cohen, G. N.; Rickenberg, H. V. (1956). Concentration spécifique réversible des amino-acides chez Escherichia coli. Ann. Inst. Pasteur, 91, 693-720.

5. Csonka, L. N. (1989). Physiological and genetic responses of bacteria to osmotic stress. Microbiol. Rev., 53, 121-147.

6. Csonka, L. N.; Epstein, W. (1996). Escherichia and Salmonella: cellular and molecular biology. In: Neidhardt, F. C., Curtis, R., Ingraham, J. L., Lin, E. C. C., Low; K. B., Magasanik, B., Reznikoff, W. S. , Riley, M., Schaechter, M. and Umbarger, H. E (eds). Osmoregulation, p. 12101223.

7. da Costa, M. S.; Santos, H.; Galinski, E. A. (1998). An overview of the 
role and diversity of compatible solutes in bacteria and archea. Advances in Biochemical Engineering/Biotechnology., 61, 117-149.

8. Galinski, E. A. (1995). Osmoadaptation in bacteria. Adv. Microb. Physiol., 37, 273-328.

9. Glaasker, E.; Konings, W. N.; Poolman, B. (1996). Osmotic regulation of intracellular solute pools in Lactococcus plantarum. J. Bacteriol., 178, 575-582.

10. Imhoff, J. F.; Rodriguez-Valera, F. (1984). Betaine is the main compatible solute of halophilic eubacteria. J. Bacteriol., 160, 478-479.

11. Jignasha, T. T.; Singh, S. P. (2007) Secretion of an alkaline protease from a salt-tolerant and alkaliphilic, Streptomyces clavuligerus strain Mit-1. Braz. J. Microbiol., 38, 766-772.

12. Kets, E. P.W.; Groot, M. N.; Galinski, E. A.; de Bont, J. A. M. (1997). Choline and acetylcholine: novel cationic osmolytes in Lactococcus plantarum. Appl. Microbiol. Biotechnol., 48, 94-98.

13. Killham, K.; Firestone, M. K. (1984a). Salt stress control of intracellular solutes in Streptomycetes indigenous to saline soils. Appl. Environ. Microbiol., 47, 301-306

14. Killham, K.; Firestone, M. K. (1984b). Proline transport increases growth efficiency in salt-stressed Streptomyces griseus. Appl. Environ. Microbiol., 48, 239-241.

15. Kushner, D.J. (1978). Life in high and solute concentrations: halophilic bacteria. In: Kushner, D.J (ed). Microbial life if extreme environments, Academic Press, Ltd, London, p. 317-368.

16. Lamosa, P.; Martins, L. O.; Da Costa, M. S.; Santos, H. (1998). Effects of temperature, salinity, and medium composition on compatible solute accumulation by Thermococcus spp. Appl. Environ. Microbiol., 64, 3591-3598.

17. Le Rudulier, D.; Bouillard, L. (1983). Glycine betaine, an osmotic effector in Klebsiella pneumoniae and other members of the Enterobacteriaceae. Appl. Environ. Microbiol., 46, 152-159.

18. Le Rudulier, D.; Mandon, K.; Dupont, L.; Trinchant, J. C. (2002). Salinity effects on the physiology of soil microorganisms. In: Bitton, G., John Wiley and Sons (eds). The encyclopedia of environmental microbiology, Inc., Hoboken, N.J, p. 2774-2789.

19. Malin, G.; Lapidot, A. (1996). Induction of synthesis of tetrahydropyrimidine derivates in Streptomyces strains and their effect on Escherichia coli in response to osmotic and heat stress. J. Bacteriol., 178, 385-395.
20. May, G.; Faatz, E.; Villagero, M.; Bremer, E. (1986). Binding protein dependant transport of glycine betaine and its osmotic regulation in Escherichia coli K 12. Mol. Gen. Genet., 205, 225-233.

21. McBride, M. J.; Ensign, J. C. (1987). Effects of intracellular trehalose content on Streptomyces griseus spores. J. Bacteriol., 169, 4995-5001.

22. Miller, E.N.; Ingram, L. O. (2007). Combined effect of betaine and trehalose on osmotic tolerance of Escherichia coli in mineral salts medium. Biotechnol. Lett., 29, 213-217.

23. Moleenar, D.; Hagting, A.; Alkema, H.; Driessen, A. J. M.; Konings, W. N. (1993). Characteristics and osmoregulatory roles of uptake systems for proline and glycine betaine in Lactococcus lactis. J. Bacteriol., 175, 5438-5444.

24. Perroud, B.; Le Rudulier, D. (1985). Glycine betaine transport in Escherichia coli: osmotic modulation. J. Bacteriol., 161, 393-401.

25. Saum, S. H.; Müller, V. (2007). Salinity-dependant switching of osmolyte strategies in a moderately halophilic bacterium: Glutamate induces proline biosynthesis in Halobacillus halophilus. J. Bacteriol., 189, 6968-6975

26. Shirling, E.B.; Gottlieb, D. (1966). Methods for characterization of Streptomyces species. Int. J. Syst. Bacteriol., 16, 313-340.

27. Singh, L.R.; Dar, T.A.; Rahman, S.; Jamal, S.; Ahmad, F. (2009). Glycine betaine may have an opposite effects on protein stability at high and low pH values. Biochimica et Biophisica Acta., 1794, 929-935.

28. Tang, S.K.; Li, W.J.; Dong, W.; Zhang, Y.G.; Xu, L.H.; Jiang, C.L. (2002). Studies of the biological characteristics of some halophilic and halotolerant actinomycetes isolated from saline and alkaline soils. Actinomycetol., 17, 06-10.

29. Vimal V.; Rajan, B. M.; Kannabira, K. (2009). Antimicrobial activity of marine actinomycete, Nocardiopsis sp. VITSVK 5 (FJ973467). Asian Journal of Medical Sciences., 1, 57-63.

30. Williams, P.G. (2009). Panning for chemical gold: marine bacteria as a source of new therapeutics. Trends Biotechnol., 27, 45-52.

31. Wu, R. Y.; Chen, M.H. (1994). Studies of the biological characteristics and halophilism of a Streptomyces strain TA4-1. Bot. Bull. Acad. Sin., $35,233-242$.

32. Xu, X.; Abo, M.; Okubo, A.; Yamazaki, S. (1998). Trehalose as osmoprotectant in Rhodobacter sphaeroides f. sp. denitrificans IL 106. Int. Biblio. Inf. Diet. Suppl., 62, 334- 337. 